\title{
Dividing Quantum Channels
}

\author{
Michael M. Wolf, J. Ignacio Cirac \\ Max-Planck-Institute for Quantum Optics, Hans-Kopfermann-Str. 1, 85748 Garching, Germany. \\ E-mail: Michael.Wolf@mpq.mpg.de
}

\begin{abstract}
We investigate the possibility of dividing quantum channels into concatenations of other channels, thereby studying the semigroup structure of the set of completely-positive trace-preserving maps. We show the existence of 'indivisible' channels which can not be written as non-trivial products of other channels and study the set of 'infinitesimal divisible' channels which are elements of continuous completely positive evolutions. For qubit channels we obtain a complete characterization of the sets of indivisible and infinitesimal divisible channels. Moreover, we identify those channels which are solutions of time-dependent master equations for both positive and completely positive evolutions. For arbitrary finite dimension we prove a representation theorem for elements of continuous completely positive evolutions based on new results on determinants of quantum channels and Markovian approximations.
\end{abstract}

\section{Contents}

I. Introduction . . . . . . . . . . . . . . . . . . . . . . 148

II. Preliminaries . . . . . . . . . . . . . . . . . . . . . . . . . . 149

III. Determinants . . . . . . . . . . . . . . . . . . 151

IV. Divisible and Indivisible Maps . . . . . . . . . . . . . . . . . . 156

V. Infinitesimal Divisible Channels . . . . . . . . . . . . . . . . . 158

VI. Qubit Channels . . . . . . . . . . . . . . . . . . 161

A Extremal qubit channels . . . . . . . . . . . . . . . . 162

B Divisible and indivisible qubit channels . . . . . . . . . . . . . . . 164

C Infinitesimal divisible qubit channels . . . . . . . . . . . . . . . . 165

VII. Conclusion . . . . . . . . . . . . . . . . . . . . . . . . . . . . . 167

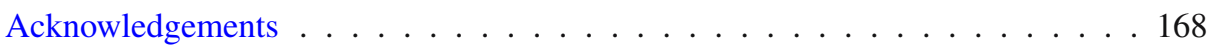

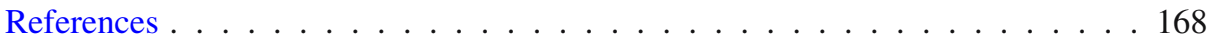




\section{Introduction}

Completely positive linear maps describe the dynamics of a quantum system in all cases where the evolution is independent of the past of the system. In the realm of quantum information theory these maps are referred to as quantum channels [1] and, clearly, the concatenation of two quantum channels is again a quantum channel. In this paper we address the converse and investigate whether and how a channel can be expressed as a non-trivial concatenation of other channels. That is, we study the semigroup structure of the set of quantum channels whose input and output systems have equal finite dimension. Despite the fact that one-parameter semigroups of completely positive maps have been extensively studied since the late sixties, the semigroup structure of the set of quantum channels as a whole appears to be widely unexplored.

The main purpose of our work is to classify the set of quantum channels with respect to (i) whether a division in terms of a concatenation is at all possible and (ii) whether a channel allows for a division into a large number of infinitesimal channels. This will lead us to the notions of divisibility and infinitesimal divisibility, where the latter property is equivalent to the existence of a continuous time-dependent completely positive evolution which has the given channel as endpoint. This classification will allow us to identify basic building blocks (generators) from which all channels can be obtained by concatenation. Furthermore, it helps us to identify those channels which are solutions of time-dependent master equations.

A graphical depiction of different notions of divisibility and their relations is given in Fig. 1. The following gives an overview on the paper and a simplified summary of the obtained results:

- Section II introduces basic results and provides a coarse but later on very useful Markovian approximation to any quantum channel.

- In Sect. III we prove some properties of the determinant of quantum channels. In particular, its strict monotonicity under concatenation, continuity bounds and properties for Kraus rank-two channels and Markovian channels.

- The notions of divisible and indivisible maps are introduced in Sect. IV. The existence of indivisible maps and generic divisibility is shown in any dimension, and it is proven that building equivalence classes under filtering operations preserves divisibility.

- Section $\mathrm{V}$ shows that every infinitesimal divisible channel can be written as a product of Markovian channels and that infinitesimal divisibility is preserved under invertible filtering operations. Equivalence to the set of continuous completely positive evolutions is proven.

- Section VI provides a complete characterization of divisible and indivisible qubit channels in terms of their Lorentz normal form. Solutions of time-dependent master equations are identified for both positive and completely positive evolutions. Channels with Kraus rank two are studied in greater detail separately.

- It is shown that already in the qubit case the vicinity of the ideal channel contains all types of channels, in particular ones that are not infinitesimal divisible and even indivisible ones.

Before going into detail we want to briefly mention some related fields and results. The notion infinite divisibility goes back to de Finetti and has thus its origin in classical probability theory where it means that for any $n \in \mathbb{N}$ a characteristic function $\chi$ is a power of another characteristic function $\chi=\chi_{n}^{n}$. Examples are the normal and Poisson distribution. Similarly, the notion of indecomposable distributions exists for those that 


\begin{tabular}{||l|}
\hline indivisible $T \neq T_{1} T_{2}$ \\
\hline divisible $T=T_{1} T_{2}$ \\
\hline infinitesimal divisible $T=\prod_{i}\left(\mathrm{id}+\epsilon_{i}\right)$ \\
\hline $\begin{array}{l}\mid \\
\text { continuous completely positive evolutions } \\
\text { time-dependent Markovian } T=\prod_{i} e^{L_{i}} \\
\text { infinitely divisible } T=T_{n}^{n} \forall n \in \mathbb{N}\end{array}$ \\
\hline Markovian $T=e^{L}$ \\
\hline
\end{tabular}

Fig. 1. Graphical depiction of the set of quantum channels regarding finer and coarser notions of divisibility, i.e, the possibility of expressing a channel in terms of a concatenation of other channels. Whereas the set of Markovian channels only contains elements of completely positive semigroups, the set of divisible channels only requires the existence of any non-trivial product decomposition. Definitions of the sets are given in the text (Sects. IV, V). Indivisible maps are discussed in Sects. IV, VI and in Sect. V it is shown that the sets of infinitesimal divisible and time-dependent Markovian channels coincide

cannot be represented as the distribution of the sum of two non-constant independent random variables.

In the 'non-commutative' context, infinite divisibility of positive matrices with respect to the Hadamard product was studied by Horn [2] and the notion was extended to quantum measurements and quantum channels by Holevo [3] and Denisov [4]. In fact, the findings of Horn can also be translated to the quantum world when considering channels with diagonal Kraus operators, as those act on a density operator by a Hadamard product with a positive matrix.

\section{Preliminaries}

This section introduces the notation and recalls some basic results which we will need in the following. Throughout we will consider linear maps $T: \mathcal{M}_{d} \rightarrow \mathcal{M}_{d}$ from the space $\mathcal{M}_{d}$ of $d \times d$ matrices into itself. It will be convenient to consider $\mathcal{M}_{d}$ as a Hilbert space $\mathfrak{H}_{d}$ equipped with the Hilbert-Schmidt scalar product $\langle A, B\rangle_{\mathfrak{H}}=\operatorname{tr}\left[A^{\dagger} B\right]$ and the 2-norm $\|A\|_{2}=\sqrt{\langle A, A\rangle_{\mathfrak{H}}} \cdot{ }^{1}$ Since $\mathfrak{H}_{d}$ is isomorphic to $\mathbb{C}^{d^{2}}$ the space of linear maps on $\mathcal{M}_{d}$ (Liouville space) is in turn isomorphic to $\mathcal{M}_{d^{2}}$. Eigenvalues and singular values of the map $T$ are then understood as the respective quantities of the matrix representation $\hat{T} \in \mathcal{M}_{d^{2}}$ of $T$. More explicit $\hat{T}_{\alpha, \beta}:=\operatorname{tr}\left[F_{\alpha}^{\dagger} T\left(F_{\beta}\right)\right]=\left\langle F_{\alpha}|T| F_{\beta}\right\rangle_{\mathfrak{H}}$, where $\left\{F_{\alpha}\right\}_{\alpha=1 \ldots d^{2}}$ is any orthonormal basis in $\mathfrak{H}_{d}$. Depending on convenience we will use three different bases: (i) matrix units $\{|i\rangle\langle j|\}_{i, j=1 \ldots d}$, (ii) generalized Gell-Mann matrices which are either diagonal or an embedding of the Pauli matrices $\sigma_{x} / \sqrt{2}$ and $\sigma_{y} / \sqrt{2}$ in $\mathcal{M}_{d}$, or (iii) a normalized unitary operator basis given by

$$
F_{\alpha}=\frac{U_{\alpha_{1}, \alpha_{2}}}{\sqrt{d}}, \quad U_{\alpha_{1}, \alpha_{2}}=\sum_{r=0}^{d-1} e^{2 \pi i r \alpha_{2} / d}\left|\alpha_{1}+r\right\rangle\langle r|, \quad \alpha_{1}, \alpha_{2}=0, \ldots, d-1 .
$$

${ }^{1}$ In general the Schatten $p$-norms are denoted by $\|A\|_{p}:=\left(\operatorname{tr}\left[|A|^{p}\right]\right)^{1 / p}$. 
When considering $T$ as a linear map on $\mathfrak{H}_{d}$ the natural norm is given by

$$
\|T\|:=\sup _{\|A\|_{2}=1}\|T(A)\|_{2}=\|\hat{T}\|_{\infty}
$$

We denote by $\mathfrak{P}$ and $\mathfrak{P}^{+}$the sets of linear maps on $\mathcal{M}_{d}$ which are positive and completely positive respectively. The corresponding subsets of trace preserving maps will be denoted by $\mathfrak{T}, \mathfrak{T}^{+}$and the elements of the latter are called channels (in the Schrödinger picture). Following Jamiolkowski's state-channel duality $[5,6]$ we can assign to every channel $T$ a state (density operator) $\tau$ by acting with $T$ on half of a maximally entangled state $\omega=\frac{1}{d} \sum_{i, j=1}^{d}|i i\rangle\langle j j|$ :

$$
\tau=(T \otimes \mathrm{id})(\omega) \text {. }
$$

The rank of this Jamiolkowski state $\tau$ (the un-normalized form of which is often called Choi matrix) is equal to the Kraus rank of $T$, i.e., the minimal number of terms in a Kraus representation [7] $T(A)=\sum_{\alpha} K_{\alpha} A K_{\alpha}^{\dagger}$. Moreover, with the involution $\left\langle i j\left|\tau^{\Gamma}\right| k l\right\rangle:=$ $\langle i k|\tau| j l\rangle$ the matrix $\tau^{\Gamma}$ leads to a matrix representation of $T$ (with matrix units as chosen basis [8]) such that

$$
\hat{T}=d \tau^{\Gamma}=\sum_{\alpha} K_{\alpha} \otimes \bar{K}_{\alpha} .
$$

By $T^{*}$ we will denote the dual of a map $T$ defined by $\operatorname{tr}\left[T^{*}(A) B\right]=\operatorname{tr}[A T(B)]$. If $T$ is trace-preserving then $T^{*}$ is unital, i.e., $T^{*}(\mathbb{1})=\mathbb{1}$ and the matrix representation corresponding to $T^{*}$ is given by the adjoint $\hat{T}^{\dagger}$.

A channel will be called Markovian if it is an element of a completely positive continuous one-parameter semigroup. That is, there exists a generator $L: \mathcal{M}_{d} \rightarrow \mathcal{M}_{d}$ with $L^{*}(\mathbb{1})=0$ such that $T_{t}=e^{t L} \in \mathfrak{T}^{+}$for all $t \geq 0$. Two equivalent standard forms for such generators were derived in [9] and [10]:

$$
\begin{aligned}
L(\rho) & =i[\rho, H]+\sum_{\alpha, \beta} G_{\alpha, \beta}\left(F_{\alpha} \rho F_{\beta}^{\dagger}-\frac{1}{2}\left\{F_{\beta}^{\dagger} F_{\alpha}, \rho\right\}_{+}\right) \\
& =i[\rho, H]+\phi(\rho)-\frac{1}{2}\left\{\phi^{*}(\mathbb{1}), \rho\right\}_{+},
\end{aligned}
$$

where $G \geq 0, H=H^{\dagger}$ and $\phi \in \mathfrak{P}^{+}$. The decomposition of the generator $L$ into a Hamiltonian part $(i[\cdot, H])$ and a dissipative part $(L-i[\cdot, H])$ becomes unique [10] if the sum in Eq. (5) runs only over traceless operators $\left(\operatorname{tr}\left[F_{\gamma}\right]=0\right)$ from an orthonormal basis in $\mathfrak{H}_{d}$ (e.g., the one in Eq. (1)). We will in the following always understand Eq. (5) in this form and call $L$ and the corresponding semigroup purely dissipative if $H=0$ w.r.t. such a representation.

Clearly, not every channel is Markovian (see [32] for necessary and sufficient conditions). However, the following lemma allows us to assign a semigroup to each channel:

Lemma 1 (Markovian approximation). For every channel $T \in \mathfrak{T}^{+}$we have that $e^{t(T-\mathrm{id})}, t \geq 0$ is a completely positive semigroup. Moreover, if $U_{0}$ is the unitary conjugation ${ }^{2}$ for which the supremum $\sup _{U} \operatorname{tr}_{\mathfrak{H}}[T U]$ is attained, then $\left(T U_{0}-\mathrm{id}\right)$ is the generator of a purely dissipative semigroup.

\footnotetext{
${ }^{2}$ By unitary conjugation we mean a channel of the form $\rho \mapsto V \rho V^{\dagger}$ with $V$ being a unitary.
} 
Proof. We will first show that $(T-\mathrm{id})$ is a valid generator by bringing it into the form of Eq. (6). Define $\phi(\rho):=\sum_{\alpha} A_{\alpha} \rho A_{\alpha}^{\dagger}$ with Kraus operators $A_{\alpha}=K_{\alpha}-x_{\alpha} \mathbb{1}$, where $\left\{K_{\alpha}\right\}$ are the Kraus operators of $T$ and $x$ is any unit vector. Then

$$
(T-\mathrm{id})(\rho)=\phi(\rho)+\kappa \rho+\rho \kappa^{\dagger},
$$

with $\kappa=\sum_{\alpha} \bar{x}_{\alpha} A_{\alpha}$. The trace preserving property $T^{*}(\mathbb{1})=\mathbb{1}$ imposes that $\phi^{*}(\mathbb{1})+$ $\kappa+\kappa^{\dagger}=0$ so that the Hermitian part of $\kappa$ is $-\phi^{*}(\mathbb{1}) / 2$. If we denote by $-i H$ with $H=H^{\dagger}$ the anti-Hermitian part then $\kappa=-\phi^{*}(\mathbb{1}) / 2-i H$ which leads to the form in Eq. (6), proving the first statement. Note that there is freedom in the choice of the anti-Hermitian part of $\kappa$ as Eq. (7) is invariant under adding to $\kappa$ a multiple of $i \mathbb{1}$.

In order to prove the second statement we have to exploit the freedom [11] in the decomposition into dissipative and Hamiltonian parts where the latter corresponds (up to multiples of $i \mathbb{1}$ ) to the anti-Hermitian part of $\kappa$. Note that

$$
\begin{aligned}
(T-\mathrm{id})(\rho) & =\sum_{\alpha}\left(A_{\alpha}-a_{\alpha} \mathbb{1}\right) \rho\left(A_{\alpha}-a_{\alpha} \mathbb{1}\right)^{\dagger}+\kappa_{a} \rho+\rho \kappa_{a}^{\dagger}, \\
\kappa_{a} & =\kappa+\sum_{\alpha} \bar{a}_{\alpha} A_{\alpha}-\mathbb{1}\left|a_{\alpha}\right|^{2} / 2 \\
& =\sum_{\alpha}\left(\bar{x}_{\alpha}+\bar{a}_{\alpha}\right)\left(K_{\alpha}-x_{\alpha} \mathbb{1}\right)-\mathbb{1}\left|a_{\alpha}\right|^{2} / 2
\end{aligned}
$$

gives other representations of the same generator for any complex vector $a$. The representation of the generator has traceless Kraus operators in Eq. (8) iff $\left(x_{\alpha}+a_{\alpha}\right)=\operatorname{tr}\left[K_{\alpha}\right] / d$. For this choice of $a$ the imaginary part of $\kappa_{a}$ in Eq. (10) would thus indeed be a multiple of $i \mathbb{1}$ if $\sum_{\alpha} \operatorname{tr}\left[\bar{K}_{\alpha}\right] K_{\alpha} \geq 0$. Let us now show that exactly this is achieved by concatenating $T$ with $U_{0}$. To this end note that by exploiting Eq. (4) we get

$$
\begin{aligned}
\operatorname{tr}_{\mathfrak{H}}\left[T U_{0}\right] & \leq \sup _{V, V^{\prime}}\left|\sum_{\alpha} \operatorname{tr} \overline{\left[K_{\alpha} V^{\prime}\right]} \operatorname{tr}\left[K_{\alpha} V\right]\right| \\
& =\sup _{V, V^{\prime}}\left|\left\langle\phi_{V^{\prime}}|\tau| \phi_{V}\right\rangle\right| \leq \sup _{V}|| \sqrt{\tau}\left|\phi_{V}\right\rangle \|^{2} \\
& =\operatorname{tr}_{\mathfrak{H}}\left[T U_{0}\right],
\end{aligned}
$$

where $V, V^{\prime}$ are unitaries and $\left|\phi_{V}\right\rangle=\sqrt{d}(V \otimes \mathbb{1}) \sum_{i=1}^{d}|i i\rangle$. On the one hand the r.h.s. of Eq. (11) is maximized if $V$ and $V^{\prime}$ are unitaries from the polar decomposition of the remaining parts, i.e., $V$ for instance is the polar unitary of $\sum_{\alpha} \operatorname{tr} \overline{\left[K_{\alpha} V^{\prime}\right]} K_{\alpha}$. On the other hand it follows from equality to $\operatorname{tr}_{\mathfrak{H}}\left[T U_{0}\right]$ that the maximum is attained for $V=V^{\prime}$ so that for $U_{0}(\cdot)=V \cdot V^{\dagger}$ we get $\sum_{\alpha} \operatorname{tr} \overline{\left[K_{\alpha} V\right]} K_{\alpha} V \geq 0$ concluding the proof.

\section{Determinants}

The multiplicativity property of determinants $\operatorname{det}\left(T_{1} T_{2}\right)=\left(\operatorname{det} T_{1}\right)\left(\operatorname{det} T_{2}\right)$ makes them an indispensable tool for the study of semigroup properties of sets of linear maps. The following theorem contains some of their basic properties. Though the results of this section are necessary for subsequent proofs they are not essential for understanding the parts on divisibility, so that this section might be skipped by the reader.

Theorem 2 (Determinants). Let $T: \mathcal{M}_{d} \rightarrow \mathcal{M}_{d}$ be a linear positive and trace preserving map. 
1. det $T$ is real and contained in the interval $[-1,1]$.

2. $|\operatorname{det} T|=1$ iff $T$ is either a unitary conjugation or unitarily equivalent to a matrix transposition.

3. If $T$ is a unitary conjugation then $\operatorname{det} T=1$, and if $\operatorname{det} T=-1$ then $T$ is a matrix transposition up to unitary equivalence. In both cases the converse holds iff $\left\lfloor\frac{d}{2}\right\rfloor$ is odd.

Proof. First note that every positive linear map satisfies $T\left(A^{\dagger}\right)=T(A)^{\dagger}$ for all $A \in \mathcal{M}_{d}$. This becomes obvious by writing $A$ as a linear combination of four positive matrices and using linearity of $T$. As a consequence all eigenvalues either come in complex conjugate pairs or are real so that det $T$ is real. From the boundedness of the norm of any trace preserving $T \in \mathfrak{T}(\|T\| \leq \sqrt{d}$ [12]) together with the fact [14] that the spectral radius equals $\lim _{m \rightarrow \infty}\left\|T^{m}\right\|^{1 / m}$ it follows that the spectral radius is one which implies $\operatorname{det} T \in[-1,1]$.

Now consider the case det $T= \pm 1$ where all eigenvalues are phases. There is always a sequence $n_{i}$ such that the limit of powers $\lim _{i \rightarrow \infty} T^{n_{i}}=: T_{\infty}$ has eigenvalues which all converge to one. ${ }^{3}$ To see that this implies that $T_{\infty}=$ id consider a two-by-two block on the diagonal of the Schur decomposition of $\hat{T}^{n_{i}}$. Up to a phase this block is of the form $\left(\begin{array}{cc}1 & c \\ 0 & e^{i \epsilon}\end{array}\right)$. Thus by taking the $p^{\text {th }}$ power of $T^{n_{i}}$ this is mapped to

$$
\left(\begin{array}{cc}
1 & \sum_{k=0}^{p-1} e^{i k \epsilon} c \\
0 & e^{i p \epsilon}
\end{array}\right) .
$$

As $\epsilon \rightarrow 0$ for $n_{i} \rightarrow \infty$ the norm $\left\|\left(T^{n_{i}}\right)^{p}\right\|$ could be increased without limit (by increasing $p$ with $n_{i}$ ) unless $c \rightarrow 0$. However, all powers of $T$ are trace preserving and positive and have therefore to have bounded norm. This rules out the survival of Jordan block-like off-diagonal elements so that $T_{\infty}=\mathrm{id}$. Hence, the inverse $T^{-1}=T_{\infty} T^{-1}=$ $\lim _{i \rightarrow \infty} T^{n_{i}-1}$ is a trace preserving positive map as well.

Assume that the image of any pure state $\Psi$ under $T$ is mixed, i.e., $T(\Psi)=\lambda \rho_{1}+(1-$ $\lambda$ ) $\rho_{2}$ with $\rho_{1} \neq \rho_{2}$. Then by applying $T^{-1}$ to this decomposition we would get a nontrivial convex decomposition for $\Psi$ (due to positivity of $T^{-1}$ ) leading to a contradiction. Hence, $T$ and its inverse map pure states onto pure states. Furthermore, they are unital, which can again be seen by contradiction. So assume $T(\mathbb{1}) \neq \mathbb{1}$. Then the smallest eigenvalue of $T(\mathbb{1})$ satisfies $\lambda_{\min }<1$ due to the trace preserving property. If we denote by $|\lambda\rangle$ the corresponding eigenvector, then $\mathbb{1}-\frac{\lambda_{\min }+1}{2} T^{-1}(|\lambda\rangle\langle\lambda|)$ is a positive operator, but its image under $T$ would no longer be positive. Therefore we must have $T(\mathbb{1})=\mathbb{1}$.

Every unital positive trace preserving map is contractive with respect to the Hilbert-Schmidt norm $[12,15]$. As this holds for both $T$ and $T^{-1}$ we have that $\forall A \in \mathcal{M}_{d}$ : $\|T(A)\|_{2}=\|A\|_{2}$, i.e., $T$ acts unitarily on the Hilbert Schmidt Hilbert space. In particular, it preserves the Hilbert Schmidt scalar product $\operatorname{tr}\left[T(A) T(B)^{\dagger}\right]=\operatorname{tr}\left[A B^{\dagger}\right]$. Applying this to pure states $A=|\phi\rangle\langle\phi|$ and $B=|\psi\rangle\langle\psi|$ shows that $T$ gives rise to a mapping of the Hilbert space onto itself which preserves the value of $|\langle\phi \mid \psi\rangle|$. By Wigner's theorem $[16,17]$ this has to be either unitary or anti-unitary. If $T$ is a unitary conjugation then $\operatorname{det} T=\operatorname{det}(U \otimes \bar{U})=1$. Since every anti-unitary is unitarily equivalent to complex conjugation, we get that $T$ is in this case a matrix transposition $T(A)=A^{T}$ (up to unitary equivalence). The determinant of the matrix transposition is easily seen in the Gell-Mann basis of $\mathcal{M}_{d}$. That is, we take basis elements $F_{\alpha}$ of the form $\sigma_{x} / \sqrt{2}, \sigma_{y} / \sqrt{2}$

\footnotetext{
3 This is an immediate consequence of Dirichlet's theorem on Diophantine approximations [13].
} 
for $\alpha=1, \ldots, d^{2}-d$ and diagonal for $\alpha=d^{2}-d+1, \ldots, d^{2}$. In this basis matrix transposition is diagonal and has eigenvalues 1 and -1 where the latter appears with multiplicity $d(d-1) / 2$. This means that matrix transposition has determinant minus one iff $d(d-1) / 2$ is odd, which is equivalent to $\left\lfloor\frac{d}{2}\right\rfloor$ being odd.

From this we get the following important corollary:

Corollary 3 (Monotonicity of the determinant). Consider the set $\mathfrak{T}$ of positive trace preserving linear maps on $\mathcal{M}_{d}$.

1. $T, T^{-1} \in \mathfrak{T}$ iff $T$ is a unitary conjugation or matrix transposition.

2. The determinant of $T \in \mathfrak{T}$ is decreasing in magnitude under composition, i.e., $|\operatorname{det} T| \geq\left|\operatorname{det} T T^{\prime}\right|$ for all $T^{\prime} \in \mathfrak{T}$ where equality holds iff $T^{\prime}$ is a unitary, a matrix transposition or $\operatorname{det} T=0$.

Part 1 of this corollary is a simple consequence of Wigner's theorem and was proven for completely positive maps for instance in [19]. ${ }^{4}$

One might wonder whether completely positive maps can have negative determinants. The following simple example answers this question in the affirmative. It is built up on the map $\rho \mapsto \rho^{T_{c}}$ which transposes the corners of $\rho \in \mathcal{M}_{d}$, i.e., $\left(\rho^{T_{c}}\right)_{k, l}$ is $\rho_{l, k}$ for the entries $(k, l)=(1, d),(d, 1)$ and remains $\rho_{k, l}$ otherwise. Note that for $d=2$ this is the ordinary matrix transposition.

Example 4. The map $T: \mathcal{M}_{d} \rightarrow \mathcal{M}_{d}$ defined by

$$
T(\rho)=\frac{\rho^{T_{c}}+1 \operatorname{tr} \rho}{1+d}
$$

is trace preserving, completely positive with Kraus rank $d^{2}-1$ and has determinant $\operatorname{det} T=-(d+1)^{1-d^{2}}$. For $d=2$ the channel is entanglement breaking and can be written as

$$
T(\rho)=\frac{1}{3} \sum_{j=1}^{6}\left|\bar{\xi}_{j}\right\rangle\left\langle\xi_{j}|\rho| \xi_{j}\right\rangle\left\langle\bar{\xi}_{j}\right|,
$$

where the six $\xi_{j}$ are the normalized eigenvectors of the three Pauli matrices.

Proof. A convenient matrix representation of the channel is given in the generalized Gell-Mann basis. Choose $F_{1}$ as the $\sigma_{y} / \sqrt{2}$ element corresponding to the corners and $F_{2}=\mathbb{1} / \sqrt{d}$ the only element which is not traceless. Then $\hat{T}=\operatorname{diag}[-1,1+d, 1, \ldots, 1] /$ $(d+1)$ leading to det $T=-(d+1)^{1-d^{2}}$.

For complete positivity we have to check positivity of the Jamiolkowski state $\tau$. The corner transposition applied to a maximally entangled state leads to one negative eigenvalue $-1 / d$. This is, however, exactly compensated by the second part of the map such that $\tau \geq 0$ with rank $d^{2}-1$.

The representation for $d=2$ is obtained from $\operatorname{tr}[A T(B)]=\operatorname{tr}\left[\left(A \otimes B^{T}\right) \tau\right] d$ by noting that in this case $\tau$ is proportional to the projector onto the symmetric subspace which in turn can be written as $\frac{1}{2} \sum_{j}\left|\xi_{j}\right\rangle\left\langle\left.\xi_{j}\right|^{\otimes 2}\right.$ in agreement with the given Kraus representation of the channel.

\footnotetext{
${ }^{4}$ It is also a consequence of [18] and therefore sometimes called the Wigner-Kadison theorem.
} 
The above example has Kraus rank $d^{2}-1$. Channels of Kraus rank two and compositions thereof can never lead to negative determinants:

Theorem 5 (Kraus rank two maps). All linear maps on $\mathcal{M}_{d}$ which are completely positive with Kraus rank two have non-negative determinant. Hence every composition of such Kraus rank two maps has non-negative determinant.

Proof. Let $A$ and $B$ be two Kraus operators of the map $T$ and assume for the moment that $\operatorname{det} A \neq 0$. Then, using matrix units as a basis for the Hilbert-Schmidt Hilbert space, we can represent the channel by the matrix $A \otimes \bar{A}+B \otimes \bar{B}$. If we use the singular value decomposition of $A=U S V$ we can write the determinant as det $T=(\operatorname{det} S)^{2 d} \operatorname{det}\left(\mathbb{1}+B^{\prime} \otimes \bar{B}^{\prime}\right)$ with $B^{\prime}=S^{-1 / 2} U^{\dagger} B V^{\dagger} S^{-1 / 2}$. Denoting the eigenvalues of $B^{\prime}$ by $b_{k}$ we obtain $\operatorname{det} T=$ $(\operatorname{det} S)^{2 d} \prod_{k, l}\left(b_{k} \bar{b}_{l}+1\right)=(\operatorname{det} S)^{2 d} \prod_{k<l}\left|b_{k} \bar{b}_{l}+1\right|^{2} \prod_{j}\left(\left|b_{j}\right|^{2}+1\right)$ which is indeed positive. As the set of maps with $\operatorname{det} A \neq 0$ is dense and $\operatorname{det} T$ continuous we obtain $\operatorname{det} T \geq 0$ for all Kraus rank two maps.

For Markovian channels the determinant can easily be expressed in terms of the dissipative part of the generator:

Theorem 6 (Determinants of Markovian Channels). Let $T=e^{L} \in \mathfrak{T}^{+}$be a Markovian channel with generator $L$ of the form in $E q$. (5). Then $\operatorname{det} T=\exp (-d \operatorname{tr}[G])$ and if $L$ is purely dissipative then $\|L\| \leq-\frac{2}{d} \ln \operatorname{det} T$.

Proof. First note that det $e^{L}=e^{\operatorname{tr} L}$, where the trace is understood in $\mathfrak{H}_{d}$. A convenient basis for computing this trace is the one in Eq. (1) as it is unitary (up to a factor) and the unitaries fulfill the Weyl relations $U_{a, b} U_{c, d}=U_{a+c, b+d} \exp (2 \pi i b c / d)$. The Hamiltonian part of the generator does not contribute to the trace. For the dissipative part assume first the basis in Eq. (5) is the one from Eq. (1) as well. Then straight forward calculation exploiting unitarity and the Weyl relations gives $\operatorname{tr}[L]=-d \operatorname{tr}[G]$. Due to the basis independence of the trace this has to be true independent of the choice of the $\left\{F_{\gamma}\right\}$ in Eq. (5) (as long as they are traceless and orthonormal).

For the second statement in the theorem we use the basis in which $G=\operatorname{diag}\left[g_{1}, g_{2}, \ldots\right]$ is diagonal:

$$
L(\rho)=\sum_{\gamma} g_{\gamma}\left(F_{\gamma} \rho F_{\gamma}^{\dagger}-\frac{1}{2}\left\{F_{\gamma}^{\dagger} F_{\gamma}, \rho\right\}_{+}\right) .
$$

From the triangle inequality together with the fact that $\left\|F_{\gamma}\right\|_{2}=1$ we obtain then $\|L\| \leq 2 \sum_{\gamma} g_{\gamma}=2 \operatorname{tr}[G]$.

Theorem 2 shows that if for a channel $\operatorname{det} T=1$, then it has to be a unitary. By continuity a channel close to a unitary will still have determinant close to one. The following is a quantitative version of the converse: if the determinant is large, then there is a unitary conjugation (namely the inverse of the one maximizing $\operatorname{tr}_{\mathfrak{H}}[T U]$ ) close to the channel. Similarly, a large determinant implies a large purity $\operatorname{tr}\left[\tau^{2}\right]$ of the Jamiolkowski state $\tau$.

Theorem 7 (Bounds on the determinant). Let $T \in \mathfrak{T}^{+}$be a channel on $\mathcal{M}_{d}$. The purity of the respective Jamiolkowski state $\tau$ leads to an upper bound on the determinant

$$
\operatorname{det} T \leq \operatorname{tr}\left[\tau^{2}\right]^{d^{2} / 2}
$$


and in the limit $\operatorname{det} T \rightarrow 1$ the distance between $T$ and the unitary conjugation $U_{0}^{-1}$ appearing in Lemma 1 vanishes as

$$
\left\|T-U_{0}^{-1}\right\|=O(\sqrt{1-\operatorname{det} T}) .
$$

Remark. An explicit however lengthy bound for the norm distance in Eq. (18) can easily be deduced from the subsequent proof.

Proof. To relate the purity to the determinant we exploit that by Eq. (4) $\hat{T}=d \tau^{\Gamma}$ is a matrix representation of the channel, so that

$$
\operatorname{tr}\left[\tau^{2}\right]=\frac{1}{d^{2}} \operatorname{tr}\left[\hat{T}^{\dagger} \hat{T}\right]=\frac{1}{d^{2}} \sum_{i=1}^{d^{2}} s_{i}^{2},
$$

where the $s_{i}$ are the singular values of $\hat{T}$ (and thus $T$ ). From this Eq. (17) is obtained via the geometric-arithmetic mean inequality together with the fact that $|\operatorname{det} T|=\prod_{i} s_{i}$.

We now use the purity bound (17) to prove the scaling in Eq. (18). The aim of the following is to relate first the purity to the largest eigenvalue of $\tau$ and then the latter to the sought distance $\left\|T-U_{0}^{-1}\right\|=\left\|T U_{0}-\mathrm{id}\right\|$.

The largest eigenvalue $\mu:=\|\tau\|_{\infty}$ of the Jamiolkowski state is for $\operatorname{tr}\left[\tau^{2}\right] \geq 1 / 2$ lower bounded by the purity via

$$
\mu \geq \frac{1}{2}\left(1+\sqrt{2 \operatorname{tr}\left[\tau^{2}\right]-1}\right) .
$$

If we denote by $\Psi=|\psi\rangle\langle\psi|$ the projector onto the eigenstate corresponding to $\mu$ then

$$
\left\|\mathbb{1} / d-\operatorname{tr}_{A} \Psi\right\|_{1} \leq\|\tau-\Psi\|_{1} \leq 2(1-\mu),
$$

where the first inequality follows from the monotonicity of the trace norm distance under the partial trace. We will now use the bounds between the trace norm distance and the fidelity [20] $f(\sigma, \rho):=\operatorname{tr} \sqrt{\rho^{1 / 2} \sigma \rho^{1 / 2}}$ :

$$
1-f(\rho, \sigma) \leq \frac{1}{2}\|\rho-\sigma\|_{1} \leq \sqrt{1-f(\rho, \sigma)^{2}} .
$$

By Uhlmann's theorem [21] we have that $f\left(\operatorname{tr}_{A} \Psi, \mathbb{1} / d\right)=\sup _{\Omega}|\langle\Omega \mid \psi\rangle|$, where the supremum is taken over all maximally entangled states $\Omega$. Denote by $\tau_{0}$ the Jamiolkowski state of $T U_{0}$ and $\left|\Omega_{0}\right\rangle=\sum_{i}|i i\rangle / \sqrt{d}$ so that $\omega=\left|\Omega_{0}\right\rangle\left\langle\Omega_{0}\right|$. Then by Eqs. (11-13) we have that $\left\langle\Omega_{0}\left|\tau_{0}\right| \Omega_{0}\right\rangle=\sup _{\Omega}\langle\Omega|\tau| \Omega\rangle$ which is in turn lower bounded by $\mu f\left(\operatorname{tr}_{A} \Psi, \mathbb{1} / d\right)^{2}$. Together with Eqs. $(21,22)$ this gives

$$
\left\langle\Omega_{0}\left|\tau_{0}\right| \Omega_{0}\right\rangle \geq \mu^{3} \text {. }
$$

Finally, we have to relate the distance between the Jamiolkowski states $\tau_{0}$ and $\omega$ to that of the respective channels. This can be done by exploiting $\operatorname{tr}[A T(B)]=\operatorname{tr}\left[\left(A \otimes B^{T}\right) \tau\right] d$ so that in general $\left\|T_{1}-T_{2}\right\|=d \sup _{A, B}\left|\operatorname{tr}\left[\left(\tau_{1}-\tau_{2}\right) A \otimes B\right]\right|$, where the supremum is taken over all operators with $\|A\|_{2}=\|B\|_{2}=1$ such that an upper bound is given by $d\left\|\tau_{1}-\tau_{2}\right\|_{1}$. In this way we get

$$
\left\|T U_{0}-\mathrm{id}\right\| \leq d\left\|\tau_{0}-\omega\right\|_{1} \leq 2 d \sqrt{1-\left\langle\Omega_{0}\left|\tau_{0}\right| \Omega_{0}\right\rangle} \leq 2 d \sqrt{1-\mu^{3}} .
$$

The scaling in Eq. (18) is then obtained by combining Eqs. $(17,20,24)$ and expanding around $\operatorname{det} T=1$. 


\section{Divisible and Indivisible Maps}

In the following we will apply the above results and study decompositions of channels in terms of concatenations of other channels, i.e., the possibility of writing $T \in \mathfrak{T}^{+}$as $T=T_{1} T_{2}, T_{i} \in \mathfrak{T}^{+}$. As the notion decomposable is commonly used in the context of convex decompositions and often refers to a specific convex decomposition of positive maps [22], we will use the notion divisible instead. Clearly, every channel is divisible in a trivial way $T=\left(T U^{-1}\right) U$, where $U$ is any unitary conjugation. In order to make the divisibility of a channel a non-trivial concept we thus define it up to unitary conjugation:

Definition 8 (Divisibility). Consider the set $\mathcal{T} \in\left\{\mathfrak{T}, \mathfrak{T}^{+}\right\}$of linear trace preserving positive or completely positive maps from $\mathcal{M}_{d}$ into itself. We say that $T \in \mathcal{T}$ is indivisible if every decomposition of the form $T=T_{1} T_{2}$ with $T_{i} \in \mathcal{T}$ is such that one of the $T_{i}$ has to be a unitary conjugation. $T$ is called divisible if it is not indivisible.

That this concept is not empty, i.e., that indivisible maps indeed exist is now a simple consequence of Thm. 2 .

Corollary 9 (Indivisible positive maps). Consider the case where $\left\lfloor\frac{d}{2}\right\rfloor$ is odd. Then the matrix transposition $\theta: \rho \mapsto \rho^{T}, \rho \in \mathcal{M}_{d}$ is indivisible within the set $\mathfrak{T}$ of positive trace preserving maps on $\mathcal{M}_{d}$.

Proof. Assume that the matrix transposition $\theta$ has a decomposition $\theta=T_{1} T_{2}$. Then by Thm. $2 \operatorname{det}\left(T_{1}\right)=-\operatorname{det} T_{2}= \pm 1$. Hence, one of the two maps has to be a transposition and the other a unitary.

Clearly, this simple observation is reminiscent of the fact that a reflection can not be expressed in terms of rotations (with positive determinant). The following is a less trivial example based on the same idea:

Corollary 10 (Indivisible completely positive maps). Consider the set $\mathfrak{T}^{+}$of completely positive trace preserving maps on $\mathcal{M}_{d}$. The channel $T_{0} \in \mathfrak{T}^{+}$with minimal determinant, i.e., for which $\operatorname{det} T_{0}=\inf _{T \in \mathfrak{T}^{+}} \operatorname{det} T$, is indivisible. For $d=2$ we have that $T_{0}(\rho)=\left(\rho^{T}+\mathbb{1}\right) / 3$ is the channel discussed in Example 4.

Proof. By Example 4 there are always channels with det $T<0$. As the set $\mathfrak{T}^{+}$of channels on $\mathcal{M}_{d}$ is compact there is always a map $T_{0}$ for which $\inf _{T \in \mathfrak{T}^{+}} \operatorname{det} T$ is attained. Now consider a decomposition $T_{0}=T_{1} T_{2}$. Then by Thm. 2 and Cor. 3 either $T_{1}$ or $T_{2}$ has to be unitary.

For the case $d=2$ recall that $T$ can be conveniently represented in terms of the real $4 \times 4$ matrix $\hat{T}_{i j}:=\operatorname{tr}\left[\sigma_{i} T\left(\sigma_{j}\right)\right] / 2$, where the $\sigma_{i}$ s are identity and Pauli matrices. In general

$$
\hat{T}=\left(\begin{array}{ll}
1 & 0 \\
v & \Delta
\end{array}\right),
$$

where $v \in \mathbb{R}^{3}, \Delta$ is a $3 \times 3$ matrix and det $\Delta=\operatorname{det} T$. To simplify matters we can diagonalize $\Delta$ by special orthogonal matrices $O_{1} \Delta O_{2}=\operatorname{diag}\left\{\lambda_{1}, \lambda_{2}, \lambda_{3}\right\}$ corresponding to unitary operations before and after the channel. Obviously, this does neither change the determinant, nor complete positivity. For the latter it is necessary that $\vec{\lambda}$ is contained in a tetrahedron spanned by the four corners of the unit cube with $\lambda_{1} \lambda_{2} \lambda_{3}=1$ 
$[23,24]$. Fortunately, all these points can indeed be reached by unital channels $(v=0)$ for which this criterion becomes also sufficient for complete positivity. By symmetry we can restrict our attention to one octant and reduce the problem to maximizing $p_{1} p_{2} p_{3}$ over all probability vectors $\vec{p}$ yielding $\vec{p}=\left(\frac{1}{3}, \frac{1}{3}, \frac{1}{3}\right)=\left(\lambda_{1}, \lambda_{2},-\lambda_{3}\right)$. Hence, the minimal determinant is $-\left(\frac{1}{3}\right)^{3}$ and the corresponding channel can easily be constructed from $\hat{T}$ as $T: \rho \mapsto \frac{1}{3}\left(\rho^{T}+\mathbb{1}\right)$.

The channels with minimal determinant lie at the border of $\mathfrak{T}^{+}$, i.e., they have reduced Kraus rank. In fact, for channels with full Kraus rank $\left(d^{2}\right)$ one can easily see that they are all divisible:

Theorem 11 (Divisibility of generic channels). If a channel $T \in \mathfrak{T}^{+}$on $\mathcal{M}_{d}$ has Kraus rank $d^{2}$, then $T$ is divisible.

Proof. Note that $T$ has full Kraus rank iff the corresponding Jamiolkowski state $\tau=$ $(T \otimes \mathrm{id})(\omega)$ has full rank. Let $T_{1} \in \mathfrak{T}^{+}$be any invertible non-unitary channel. Then $\left(T_{1}^{-1} T \otimes \mathrm{id}\right)(\omega)$ is still positive if only $T_{1}$ is sufficiently close to the identity. Therefore $T_{2}:=T_{1}^{-1} T$ is an admissible channel so that $T=T_{1} T_{2}$. Clearly, one can always choose $T_{1}$ such (e.g. of Kraus rank two, or det $T_{1} \neq \operatorname{det} T$ ) that neither $T_{1}$ nor $T_{2}$ are unitary.

We will now see that in searching for a decomposition of a trace preserving map $T=T_{1} T_{2}$ we can essentially drop the trace preserving constraint on $T_{1}$ and $T_{2}$. That is, if there exists a non-trivial decomposition into non-trace preserving maps, then there will be one in terms of trace preserving maps as well:

Theorem 12. Let $\mathcal{P} \in\left\{\mathfrak{P}, \mathfrak{P}^{+}\right\}$be either the set of positive or completely positive linear maps on $\mathcal{M}_{d}$, and $\mathcal{T} \in\left\{\mathfrak{T}, \mathfrak{T}^{+}\right\}$the respective subset of trace preserving maps. Then for every concatenation $\tilde{T}_{1} \tilde{T}_{2}=T, \tilde{T}_{i} \in \mathcal{P}, T \in \mathcal{T}$ with $\operatorname{det} \tilde{T}_{1}^{*}(\mathbb{1}) \neq 0$ there exist $T_{1}, T_{2} \in \mathcal{T}$ with Kraus $\operatorname{rank}^{5} \operatorname{rank}\left[T_{i}\right]=\operatorname{rank}\left[\tilde{T}_{i}\right]$ such that $T_{1} T_{2}=T$.

Proof. We will explicitly construct $T_{1}$ and $T_{2}$ via their duals. Due to positivity and the absence of a kernel in $\tilde{T}_{1}^{*}(\mathbb{1})$ we can find a positive definite matrix $P>0$ which is the square root of $\tilde{T}_{1}^{*}(\mathbb{1})=P^{2}$. Then $T_{1}^{*}(X):=P^{-1} \tilde{T}_{1}^{*}(X) P^{-1}$ fulfills $T_{1}^{*}(\mathbb{1})=\mathbb{1}$ and is thus the dual of a map $T_{1} \in \mathcal{T}$. Defining $T_{2}^{*}(X):=\tilde{T}_{2}^{*}(P X P)$ we obtain $T_{2}^{*} T_{1}^{*}=T^{*}$ so that indeed $T_{1} T_{2}=T$. Moreover, $T_{2} \in \mathcal{T}$ since $T_{2}^{*}(\mathbb{1})=T_{2}^{*} T_{1}^{*}(\mathbb{1})=T^{*}(\mathbb{1})=\mathbb{1}$. Equality for the Kraus ranks follows immediately from the fact that $T_{i}$ and $\tilde{T}_{i}$ differ merely by concatenation with an invertible completely positive Kraus rank-one map.

For the classification of (in-)divisible maps this allows us to restrict to equivalence classes under invertible filtering operations. In Sect. VI this reduction will enable us to completely characterize the set of indivisible qubit channels.

Corollary 13 (Reduction to normal form). Let $\mathcal{T} \in\left\{\mathfrak{T}, \mathfrak{T}^{+}\right\}$and $T, \tilde{T} \in \mathcal{T}$ be related via $T=T_{A} \tilde{T} T_{B}$ where $T_{A}, T_{B} \in \mathfrak{P}^{+}$are invertible completely positive maps with Kraus rank one. Then $T$ is divisible iff $\tilde{T}$ is divisible.

\footnotetext{
5 For positive maps we define the Kraus rank as the rank of the corresponding Jamiolkowski operator (Choi matrix).
} 


\section{Infinitesimal Divisible Channels}

In this section we will refine the somewhat coarse notion of divisibility by asking which channels can be broken down into infinitesimal pieces, i.e., into channels arbitrary close to the identity. This will lead us to a number of a priori different sets of channels, depending on the additional structure which we impose on the infinitesimal constituents. The main result will then be the equivalence of three of these sets, showing that the imposed structure is not an additional requirement but rather emerges naturally.

Let us begin with the most structured and best investigated of these sets: the set of Markovian channels. Evidently, a Markovian channel, i.e., an element of a continuous completely positive one-parameter semigroup is divisible. Furthermore it can be divided into a large number of equal infinitesimal channels and it is the solution of a time-independent master equation

$$
\frac{\partial \rho}{\partial t}=L(\rho)
$$

with $L$ of the form in Eqs. $(5,6)$.

Following the terminology used in classical probability theory one calls a channel $T$ infinitely divisible [1,4] if for all $n \in \mathbb{N}$ there is another channel $T_{n}$ such that $T=T_{n}^{n}$. It was shown in [4] that infinitely divisible channels are all of the form $T=T_{0} e^{L}$, where $L$ is a Lindblad generator of the form in Eq. (5) and $T_{0}$ is an idempotent channel satisfying $T_{0} L=T_{0} L T_{0}$. Hence, an infinitely divisible channel becomes an element of a continuous completely positive one-parameter semigroup if $T_{0}=\mathrm{id}$.

Consider now a more general family, which one might refer to as continuous completely positive evolutions. That is, for some time interval $[0, t]$ there exists a continuous mapping $[0, t] \times[0, t] \rightarrow \mathfrak{T}^{+}$onto a family of quantum channels $\left\{T\left(t_{2}, t_{1}\right)\right\}$ such that

1. $T\left(t_{3}, t_{2}\right) T\left(t_{2}, t_{1}\right)=T\left(t_{3}, t_{1}\right)$ for all $0 \leq t_{1} \leq t_{2} \leq t_{3} \leq t$,

2. $\lim _{\epsilon \rightarrow 0}\|T(\tau+\epsilon, \tau)-\mathrm{id}\|=0$ for all $\tau \in[0, t)$.

In other words there is a continuous path within $\mathfrak{T}^{+}$which connects the identity with each element of this family and along which we can move (one-way) by concatenation with quantum channels. Let us denote by $\mathcal{J} \subset \mathfrak{T}^{+}$the set of all elements of such continuous completely positive evolutions. Clearly, this set is included in the following:

Definition 14 (Infinitesimal divisibility). Define a set $\mathcal{I}$ of channels $T \in \mathfrak{T}^{+}$with the property that for all $\epsilon>0$ there exists a finite set of channels $T_{i} \in \mathfrak{T}^{+}$such that (i) $\left\|T_{i}-\mathrm{id}\right\| \leq \epsilon$ and (ii) $\prod_{i} T_{i}=T$. We say that a channel is infinitesimal divisible if it belongs to the closure $\overline{\mathcal{I}}$.

Remark. Note that every infinitely divisible channel is also infinitesimal divisible. To see this note that for every idempotent channel $T_{0}$ we have that $\left[(1-\epsilon) \mathrm{id}+\epsilon T_{0}\right]^{n}$ is a product of channels which are $\epsilon$-close to the identity with convergence to $T_{0}$ for $n \rightarrow \infty$.

By continuity and multiplicativity of the determinant we obtain a simple necessary condition for a channel to be infinitesimal divisible:

Proposition 15. If a channel $T \in \mathfrak{T}^{+}$is infinitesimal divisible, then $\operatorname{det} T \geq 0$.

A similar notion of infinitesimal divisibility can be defined by introducing a set $\mathcal{I}^{\prime}$ analogous to the set $\mathcal{I}$ with the additional restriction that all the $T_{i} \in \mathfrak{T}^{+}$have to be Markovian, i.e., of the form $T_{i}=e^{L_{i}}$ with $L_{i}$ a Lindblad generator. Clearly, $\overline{\mathcal{I}^{\prime}} \subseteq \overline{\mathcal{I}}$ and 
intuitively the converse should also hold as every channel close to the identity should be 'almost Markovian'. However, the closer the $T_{i}$ are to the identity, the more terms we need in the product $\prod_{i}^{n} T_{i}=T$. Hence, $n$ will be an increasing function of $\epsilon$ and the question whether or not one can safely replace each $T_{i}$ by a Markovian channel amounts to the estimation of an accumulated error of the form " $n \epsilon$ ". The following theorem shows that the scaling of the latter is benign so that indeed $\overline{\mathcal{I}}=\overline{\mathcal{I}^{\prime}}$. Moreover, since $\overline{\mathcal{I}^{\prime}} \subseteq \overline{\mathcal{J}} \subseteq \overline{\mathcal{I}}$ both sets are equal to the set of continuous completely positive evolutions.

Theorem 16 (Structure of infinitesimal divisible channels). With the above notation we have that $\overline{\mathcal{I}}=\overline{\mathcal{I}^{\prime}}=\overline{\mathcal{J}}$. In particular, every infinitesimal divisible channel can be arbitrary well approximated by a product of Markovian channels.

Proof. We want to show that one can replace every channel $T_{i}$ in the decomposition $T=\prod_{i=1}^{n} T_{i}$ with $\left\|T_{i}-\mathrm{id}\right\| \leq \epsilon$ by a Markovian channel such that the error becomes negligible in the limit $\epsilon \rightarrow 0$ (and thus $n \rightarrow \infty$ ). This is proven in two steps: (i) we calculate the error obtained from the Markovian approximation in Lem. 1 as a function of $n$ and $\epsilon$, and (ii) we relate $n$ and $\epsilon$ by exploiting properties of the determinant shown in Thms. 6, 7. Strictly speaking, we will in both steps not use the distance $\epsilon$ to the identity but rather a distance $\delta \leq \epsilon$ to a nearby unitary.

First we write $\prod_{i} \bar{T}_{i}=\prod_{i} \tilde{T}_{i} U_{i}$, where $\tilde{T}_{i}=T_{i} U_{i}^{-1}$ is such that $\left(\tilde{T}_{i}-\right.$ id) is a purely dissipative generator according to Lem. 1 . The idea is then to approximate $\tilde{T}_{i}$ by $\exp \left(\tilde{T}_{i}-\mathrm{id}\right)$. The total error in this approximation is then given by

$$
\left\|\prod_{i} T_{i}-\prod_{i} e^{\tilde{T}_{i}-\mathrm{id}} U_{i}\right\|=\left\|\prod_{i} T_{i}-\prod_{i}\left(T_{i}+\Delta_{i}\right)\right\|,
$$

where $\Delta_{i}=\left(\exp \left[\tilde{T}_{i}-\mathrm{id}\right]-\tilde{T}_{i}\right) U_{i}$ is an operator whose norm vanishes as $O\left(\left\|\tilde{T}_{i}-\mathrm{id}\right\|^{2}\right)$. The product $\prod_{i}\left(T_{i}+\Delta_{i}\right)$ contains $\left(\begin{array}{l}n \\ k\end{array}\right)$ terms of the form " $T_{i}^{n-k} \Delta_{i}^{k}$ " where the $T_{i}$ s come in at most $k+1$ groups for each of which we can bound the norm by $\sqrt{d}$ [12]. If we define $\delta:=\max _{i}\left\|\tilde{T}_{i}-\mathrm{id}\right\|$ we can therefore bound the error in Eq. (27) by

$$
\left\|\prod_{i} T_{i}-\prod_{i}\left(T_{i}+\Delta_{i}\right)\right\| \leq \sqrt{d}\left(\left(1+\sqrt{d} O\left(\delta^{2}\right)\right)^{n}-1\right) .
$$

This vanishes iff $\delta^{2} n \rightarrow 0$ as $n \rightarrow \infty .^{6}$

To relate $\delta$ and $n$ we use Thm. 6 from which we obtain $\delta \leq-\frac{2}{d} \min _{i} \ln \operatorname{det} \exp \left(\tilde{T}_{i}-\right.$ id). Exploiting continuity of the determinant ${ }^{7}$ and denoting by $\tilde{T}_{\delta}$ the channel $T_{i}$ giving rise to the maximum distance $\delta$, this gives

$$
\delta \leq-\frac{2}{d} \ln \left[\operatorname{det} \tilde{T}_{\delta}-O\left(\delta^{2}\right)\right] .
$$

Since by assumption there are arbitrarily fine-grained decompositions $T=\prod_{i} T_{i}$ we can w.l.o.g. assume that all $T_{i}$ have equal determinant $\operatorname{det} T_{i}=(\operatorname{det} T)^{1 / n}$ (or ones distributed within a sufficiently narrow interval). As $\operatorname{det} \tilde{T}_{i}=\operatorname{det} T_{i}$ Eq. (29) relates $n$ and

${ }^{6}$ An alternative way for obtaining this result is by defining $C(l):=\prod_{i=1}^{l} T_{i} \prod_{j=l+1}^{n}\left(T_{j}+\Delta_{j}\right)$. Then Eq. (27) equals $\|C(n)-C(0)\|=\left\|\sum_{k=0}^{n-1} C(k+1)-C(k)\right\| \leq(n+1) d \delta^{2}$, where the inequality follows from the triangle inequality.

${ }^{7}|\operatorname{det} A-\operatorname{det} B| \leq d\|A-B\| \max \{\|A\|,\|B\|\}^{d-1}[14]$. 
$\delta$ - unfortunately in a way that we cannot yet conclude that $\delta=o\left(n^{-1 / 2}\right)$. However, it enables us to lift any polynomial bound to higher order: assume that $\delta=O\left(n^{-q}\right)$ for some $q \in(0,1)$. Then Eq. (29) gives rise to $\delta=O\left(n^{-2 q}-(\ln \operatorname{det} T) / n\right)$ which leads recursively to $\delta=O(1 / n)$ provided that $\operatorname{det} T>0$. Hence, any bound of the form $\delta=O\left(n^{-q}\right), q>0$ will suffice to show that the error given by Eq. (27) vanishes asymptotically. Such a bound is provided by Thm. 7 as we obtain from Eq. (18) that $\delta=O(\sqrt{-(\ln \operatorname{det} T) / n})$.

Note finally that it suffices to consider the case det $T>0$ as singular channels are only included in Def. 8 by taking the closure of $\mathcal{I}$ and det $T<0$ is excluded by Prop. 15 .

Similar to the notion of divisibility we may introduce infinitesimal divisible positive maps by replacing $\mathfrak{T}^{+}$in Def. 14 by $\mathfrak{T}$. In both cases we can again decide whether a map is infinitesimal divisible by considering its normal form under invertible filtering operations with Kraus rank one:

Theorem 17 (Reduction to normal form). Let $\mathcal{T} \in\left\{\mathfrak{T}, \mathfrak{T}^{+}\right\}$and $T, \tilde{T} \in \mathcal{T}$ be related via $T=T_{A} \tilde{T} T_{B}$, where $T_{A}, T_{B} \in \mathfrak{P}^{+}$are invertible completely positive maps with Kraus rank one. Then $T$ is infinitesimal divisible iff $\tilde{T}$ is.

Proof. As the statement is symmetric in $T$ and $\tilde{T}$ (due to invertibility of $T_{A}, T_{B}$ ) it is sufficient to prove one direction. So let us assume that $\tilde{T}=\prod_{i=1}^{n} \tilde{T}_{i}$ is infinitesimal divisible. Then we can write $T=\prod_{i=1}^{n} R_{i} \tilde{T}_{i} R_{i+1}^{-1}$, where $R_{i} \in \mathfrak{P}^{+}$are invertible maps of Kraus rank one with $R_{1}=T_{A}$ and $R_{n+1}^{-1}=T_{B}$. We will now show that the intermediate $R_{i}$ 's can be chosen such that $T_{i}:=R_{i} \tilde{T}_{i} R_{i+1}^{-1} \in \mathcal{T}$ is such that $\left\|T_{i}-\mathrm{id}\right\|$ vanishes uniformly as $\left\|\tilde{T}_{i}-\mathrm{id}\right\| \leq \epsilon \rightarrow 0$. This is achieved by recursively constructing $R_{i+1}$ from $R_{i}$ according to the proof of Thm. 12 and exploiting that

$$
\left\|T_{i}-\mathrm{id}\right\| \leq\left\|R_{i} R_{i+1}^{-1}-i d\right\|+\epsilon\left\|R_{i}\right\|\left\|R_{i+1}^{-1}\right\| .
$$

Let us denote by $K_{i}=U_{i} P_{i}$ the polar decomposition of the Kraus operator of $R_{i}(\cdot)=$ $K_{i} \cdot K_{i}^{\dagger}$. The trace preserving requirement for $T_{i}$ imposes that $R_{i+1}^{-1 *} \tilde{T}_{i}^{*} R_{i}^{*}(\mathbb{1})=\mathbb{1}$ which is achieved by choosing $P_{i+1}=\sqrt{\tilde{T}_{i}^{*}\left(P_{i}^{2}\right)}$. As any unital positive map is spectrumwidth decreasing [19] we have for the range of eigenvalues $\left[\lambda_{\min }\left(P_{i+1}\right), \lambda_{\max }\left(P_{i+1}\right)\right] \subseteq$ $\left[\lambda_{\min }\left(P_{i}\right), \lambda_{\max }\left(P_{i}\right)\right]$. This allows us to bound the second term in Eq. (30) by $\epsilon\left\|R_{i}\right\|$ $\left\|R_{i+1}^{-1}\right\| \leq \epsilon \lambda_{\text {max }}^{2}\left(P_{1}\right) \lambda_{\text {min }}^{-2}\left(P_{1}\right)$.

To bound the first term note that $\left\|\tilde{T}_{i}^{*}\left(P_{i}^{2}\right)-P_{i}^{2}\right\| \leq \epsilon\left\|P_{i}^{2}\right\|_{2} \leq \epsilon \lambda_{\max }^{2}\left(P_{i}\right) \sqrt{d}$. By continuity of the square $\operatorname{root}^{8}$ this implies $\left\|P_{i+1}-P_{i}\right\| \leq \sqrt{\epsilon} d^{1 / 4} \lambda_{\text {max }}\left(P_{i}\right)$. Hence, $\left\|P_{i} P_{i+1}^{-1}-\mathbb{1}\right\| \leq \sqrt{\epsilon} d^{1 / 4} \lambda_{\max }\left(P_{i}\right) \lambda_{\text {min }}^{-1}\left(P_{i+1}\right)$ yielding a $\sqrt{\epsilon}$ bound for the first term in Eq. (30) if we take $U_{i+1}=U_{i}$. The latter choice might not be possible in the $n^{\text {th }}$ step (as the trace preserving requirement only fixes $T_{B}$ up to a unitary conjugation). However, we can always add an additional unitary without changing the property of being infinitesimal divisible.

Note that the above reduction to normal form together with Thm. 16 preserves continuity in the sense that if $T=T_{A} e^{L} T_{B}$ with Markovian $e^{L} \in \mathfrak{T}^{+}$, then we can write

$$
T=\mathbb{T} e^{\int_{0}^{1} L(\tau) d \tau}
$$

${ }^{8}\|\sqrt{A}-\sqrt{B}\| \leq\|A-B\|^{1 / 2}$ for all positive $A, B[14]$. 
where $\mathbb{T}$ is the time-ordering operator and $\tau \mapsto L(\tau)$ is a continuous mapping onto generators of the form in Eqs. $(5,6)$. In other words, $T$ is then a solution of a timedependent master equation $d \rho / d t=L(t) \rho$. The fact that every generic infinitesimal divisible channel can be written in this way is proven below for the case $d=2$ of qubit channels.

\section{Qubit Channels}

The simplicity of qubit channels $\left(T: \mathcal{M}_{2} \rightarrow \mathcal{M}_{2}\right)$ often allows a more thorough analysis of their properties. An exhaustive investigation of the convex structure of the set of qubit channels and positive trace-preserving qubit maps was for instance given in [25] and [26] respectively. Similarly, their normal form under invertible filtering operations was determined in [27]. In the following we will make extensive use of these results in order to derive a complete characterization of the above discussed semigroup structure of this set. We begin by recalling some of the basic tools and treat the case of extremal qubit channels (two Kraus operators) first, as later argumentation will build on this. The main results - a complete characterization of divisible and infinitesimal divisible qubit channels - are then stated in Thm. 23 and Thm. 24.

The representation we will mainly use in the following is a real $4 \times 4$ matrix $\hat{T}_{i j}:=$ $\operatorname{tr}\left[\sigma_{i} T\left(\sigma_{j}\right)\right] / 2$ (cf. [25]) which is in turn characterized by a $3 \times 3$ block $\Delta$ and a vector $v \in \mathbb{R}^{3}$ encoding the correlations and the reduced density matrix of the Jamiolkowski state respectively:

$$
\hat{T}=\left(\begin{array}{ll}
1 & 0 \\
v & \Delta
\end{array}\right) .
$$

Since there is an epimorphism from $S U$ (2) to the rotation group $S U$ (3) we can always diagonalize $\Delta$ by acting unitarily before and after $T$. More specifically, for any $T \in \mathfrak{T}$ there exist unitary conjugations $U_{1}, U_{2}$ such that $U_{1} T U_{2}$ has $\Delta=\operatorname{diag}\left(\lambda_{1}, \lambda_{2}, \lambda_{3}\right)$ with $1 \geq \lambda_{1} \geq \lambda_{2} \geq\left|\lambda_{3}\right|$. Expressing complete positivity in terms of $v$ and $\lambda$ is rather involved and discussed in detail in [23-25]. A necessary condition for complete positivity is that

$$
\lambda_{1}+\lambda_{2} \leq 1+\lambda_{3}
$$

which becomes sufficient if the channel is unital, i.e., $v=0$.

A very useful standard form for qubit channels is obtained when building equivalence classes under filtering operations [27]. ${ }^{9}$

Theorem 18 (Lorentz normal form). For every qubit channel $T \in \mathfrak{T}^{+}$there exist invertible $T_{A}, T_{B} \in \mathfrak{P}^{+}$, both of Kraus rank one, such that $T_{A} T T_{B}=\tilde{T} \in \mathfrak{T}^{+}$is of one of the following three forms:

1. Diagonal: $\tilde{T}$ is unital ( $v=0)$. This is the generic case.

2. Non-diagonal: $\tilde{T}$ has $\Delta=\operatorname{diag}(x / \sqrt{3}, x / \sqrt{3}, 1 / 3), 0 \leq x \leq 1$ and $v=(0,0,2 / 3)$. These channels have Kraus rank 3 for $x<1$ and Kraus rank 2 for $x=1$.

3. Singular: $\tilde{T}$ has $\Delta=0$ and $v=(0,0,1)$. This channel has Kraus rank 2 and is singular in the sense that it maps everything onto the same output.

\footnotetext{
9 This standard form is referred to as Lorentz normal form as the mapping $T \mapsto T_{A} T T_{B}$ corresponds to $\hat{T} \mapsto L_{A} \hat{T} L_{B}$, where $L_{A, B}$ are proper orthochronous Lorentz transformations [27].
} 
A concatenation of qubit channels $T_{1} T_{2}=T$ corresponds to a multiplication of the respective matrices $\hat{T}_{1} \hat{T}_{2}=\hat{T}$ so that $\Delta_{1} \Delta_{2}=\Delta$ and $\Delta_{1} v_{2}+v_{1}=v$. In this way we can for instance decompose every channel of the second form in Thm. 18 into

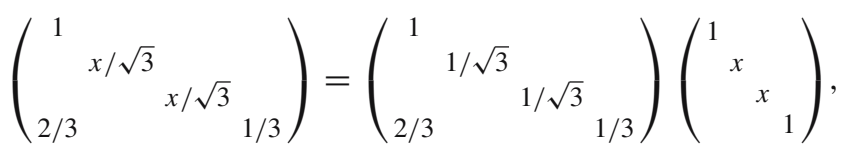

which is a concatenation of two Kraus rank-two channels (unless $x=1$ where the initial channel is already rank-two). Let us now have a closer look at qubit channels with Kraus rank two.

A. Extremal qubit channels. Channels with Kraus rank two play an important role regarding the convex structure of the set of qubit channels. It was shown in [25] that every extreme point of this set is either a unitary conjugation or a (non-unital) Kraus rank-two channel. In this context it has been shown that every Kraus rank-two channel can up to unitary conjugations be represented by

$$
\hat{T}=\left(\begin{array}{cccc}
1 & & & \\
& c_{u} & & \\
& & c_{v} & \\
s_{u} s_{v} & & & c_{u} c_{v}
\end{array}\right), \quad c_{u}=\cos u, s_{u}=\sin u .
$$

For the remainder of this subsection we will, however, use a different representation which is very handy for our purposes albeit less explicit than the one in Eq. (35). This will allow us to prove the following:

Theorem 19 (Infinitesimal divisibility of Kraus rank-two channels). Let $T: \mathcal{M}_{2} \rightarrow$ $\mathcal{M}_{2}$ be a qubit channel with Kraus rank two. Then there exist unitary conjugations $U_{1}, U_{2}$, a continuous time-dependent Lindblad generator $L$ and $t>0$ such that

$$
U_{1} T U_{2}=\mathbb{T} e^{\int_{0}^{t} L(\tau) d \tau} .
$$

In order to prove this result, we will first introduce the mentioned normal form and then explicitly construct the Lindblad generators. To this end consider the set of specific channels $C \in \mathfrak{T}^{+}$with Kraus operators

$$
\begin{aligned}
& A_{1}=|0\rangle\langle a|, \\
& A_{2}=|0\rangle\langle b|+x| 1\rangle\langle 1| .
\end{aligned}
$$

We will take $x$ and the zero components of $|a\rangle$ and $|b\rangle$ real. The trace preserving condition gives

$$
|a\rangle\langle a|+| b\rangle\left\langle b\left|=\mathbb{1}-x^{2}\right| 1\right\rangle\langle 1| .
$$

We will prove that all channels $C$ are of the form on the r.h.s. of Eq. (36), which, together with the following lemma, will yield the proof of the theorem.

Lemma 20. For any qubit channel $T$ with Kraus rank two, there exist unitary conjugations $U_{1}, U_{2}$ such that $T=U_{1} C U_{2}$. 
Proof. Given the Kraus operators $K_{1,2}$ of $T$, we can always find $\alpha_{1,2}$ such that $\alpha_{1} K_{1}+$ $\alpha_{2} K_{2}$ has rank 1 (i.e., zero determinant). Thus, a different set of Kraus operators can be chosen with $\hat{K}_{1}=\left|e_{0}\right\rangle\left\langle f_{1}\right|$, and $\hat{K}_{2}=\left|e_{0}\right\rangle\left\langle f_{2}|+| e_{1}\right\rangle\left\langle f_{3}\right|$, where $e_{0,1}$ are orthonormal. Defining $A_{i}=V_{1} \hat{K}_{i} V_{2}$, with $V_{1}, V_{2}$ unitaries, using the fact that we can multiply Kraus operators with complex numbers of unit modulus, and imposing that the channel is trace preserving, we easily reach the above form.

Thus, from now on we concentrate on the specific channels $C$. Depending on the vectors $a, b$, we can have very different channels. We define:

Definition 21. Given a channel of the above form $C$, we will call it: (i) class-1 if $\langle a \mid 0\rangle=$ $\langle b \mid 1\rangle=0$; (ii) class-2 if it is not in class-1 and $x=1$; (iii) class-3 otherwise.

The main difference between these channels lies on the number of pure states that are mapped into pure states. In fact, it can be easily checked that for all channels $|0\rangle \rightarrow|0\rangle$ and that for class- 1 channels, either all pure states are mapped into $|0\rangle$ (for $x=0$ ) or only $|0\rangle$ is mapped into a pure state (for $x \neq 0$ ), whereas for class-2 and 3, apart from $|0\rangle$, there is only one state $|c\rangle \perp|a\rangle$ which is mapped into a pure state. In the following we will consider the different classes of channels independently.

a. Class- 1 channels. We can write $|a\rangle=\left(1-x^{2}\right)^{1 / 2}|1\rangle$ and $|b\rangle=|0\rangle$, so that all these channels are parametrized just by $x$, and therefore we will write $C_{x}$. We have

$$
C_{x_{1}} C_{x_{2}}=C_{x_{1} x_{2}}, \quad C_{1}=1
$$

Thus, this class forms a continuous 1-parameter semigroup. Using infinitesimal transformations one can easily show that

$$
C_{x}=e^{-\ln (x) L}, \quad L(\rho)=2|0\rangle\langle 1|\rho| 1\rangle\langle 0|-\rho| 1\rangle\langle 1|-| 1\rangle\langle 1| \rho .
$$

b. Class-2 channels. In this case we can write $|a\rangle=\left(1-y^{2}\right)^{1 / 2}|0\rangle$ and $|b\rangle=y|0\rangle$, so that again we have a single parameter family $C_{y}$. As before, we obtain a one-parameter semigroup $C_{y}=\exp (-\ln (y) L)$ but now with $L(\rho)=2 \sigma_{z} \rho \sigma_{z}-2 \rho$.

c. Class-3 channels. We show now that every channel $C$ in this class is completely determined by the vector different from $|0\rangle$ which is mapped into a pure state.

As mentioned above, this class is characterized by the fact that a normalized pure state $|c\rangle \perp|a\rangle$ is mapped into another pure $\left|c^{\prime}\right\rangle$ :

$$
\begin{aligned}
|c\rangle & =c_{0} e^{i \varphi}|0\rangle+c_{1}|1\rangle, \quad c_{0}, c_{1} \in \mathbb{R}, \\
\left|c^{\prime}\right\rangle & =y c_{0} e^{i \varphi}|0\rangle+x c_{1}|1\rangle,
\end{aligned}
$$

where $y \geq 1$ ensures normalization. That is, since $x<1$, the distance to the vector $|0\rangle$ decreases, whereas the azimuthal angle in the Bloch sphere remains constant. Now we will show the converse:

Lemma 22. Given $|c\rangle$ and $\left|c^{\prime}\right\rangle$ as in Eq. (41) with $x<1$, there exists a unique class-3 channel which maps $|c\rangle \rightarrow\left|c^{\prime}\right\rangle$. 
Proof. The definition of $c$ and $c^{\prime}$ fixes the values of $x$ and $|a\rangle$ up to a normalization for the Kraus operators (37). Both $\|a\|$ and $|b\rangle$ are completely specified by the condition (38). Indeed, defining $|\tilde{a}\rangle:=|a\rangle /|| a||$ we have to fulfill that $\mathbb{1}-x^{2}|1\rangle\left\langle 1|-\| a|^{2} \mid \tilde{a}\right\rangle\langle\tilde{a}|=$ $|b\rangle\langle b|$, i.e., has rank 1 , which automatically fixes

$$
\|a\|^{2}=\frac{1-x^{2}}{1-x^{2} c_{1}^{2}}
$$

and thereby $|b\rangle$ through Eq. (38).

The maps in this class are parametrized by $x, c_{1} \in(0,1)$ and $\varphi \in[0,2 \pi)$, and thus we will write $C_{c_{1}, x, \varphi}$. They fulfill

$$
C_{x c_{1}, y, \varphi} C_{c_{1}, x, \varphi}=C_{c_{1}, x y, \varphi} .
$$

Note that $C_{c_{1}, x, \varphi} \rightarrow$ id for $x \rightarrow 1$. Thus, we can determine the generator of an infinitesimal transformation as $L_{c_{1}, \varphi}:=\lim _{\epsilon \rightarrow 0}\left(\mathrm{id}-C_{c_{1}, e^{-\epsilon, \varphi}}\right) / \epsilon$. We obtain

$$
\begin{aligned}
L_{c_{1}, \varphi}(\rho) & =i\left[\rho, H_{c_{1}, \varphi}\right]+D_{c_{1}, \varphi}(\rho), \\
H_{c_{1}, \varphi} & =\frac{c_{1}}{i c_{0}}\left(e^{i \varphi}|0\rangle\left\langle 1\left|-e^{-i \varphi}\right| 1\right\rangle\langle 0|\right),
\end{aligned}
$$

and $D_{c_{1}, \varphi}$ is a simple dissipative Lindblad generator characterized by a single Kraus operator of the form $A_{c_{1}, \varphi}=\sqrt{2}|0\rangle\left(c_{1}\langle 0|-c_{0}\langle 1|\right) / c_{0}$, with $c_{0}=\left(1-c_{1}^{2}\right)^{1 / 2}$. Thus, we arrive at the result of Thm. 19 and can write

$$
C_{c_{1}, x, \varphi}=\mathbb{T} \exp \int_{0}^{-\ln (x)} L_{c_{1} e^{-\tau}, \varphi} d \tau .
$$

B. Divisible and indivisible qubit channels. We are now prepared to give a complete characterization of divisible/indivisible qubit channels. An indivisible example-the channel with minimal determinant_was already given in Corollary 10. Surprisingly, there are indivisible channels with positive determinant as well:

Theorem 23 (Indivisible qubit channels). A non-unitary qubit channel is indivisible within $\mathfrak{T}^{+}$if and only if it has Kraus rank three and its Lorentz normal form (Thm. 18) is diagonal (i.e., unital).

Proof. As all qubit channels with Kraus rank four are divisible due to Thm. 11 and all rank-two channels are divisible according to the previous subsection, the Kraus rank of indivisible qubit channels must be three (or one-trivially). Following Cor. 13 it suffices to consider the Lorentz normal form of Thm. 18. Since the non-diagonal case can be decomposed via Eq. (34) into divisible Kraus rank-two channels, it remains to show that all unital channels with Kraus rank three are indivisible.

Suppose $T$ is such a channel and we can write $T=T_{1}^{\prime} T_{2}^{\prime}$ with non-unitary $T_{i}^{\prime} \in \mathfrak{T}^{+}$. Then there is also a decomposition $T=T_{1} T_{2}$ into non-unitary unital channels $T_{i}$ which can for instance be obtained by setting the $v$ 's in $\hat{T}_{i}^{\prime}$ in Eq. (32) to zero and keeping $\Delta_{i}=\Delta_{i}^{\prime}$. This will still be a decomposition of $T$ but neither change the determinant (and thus non-unitarity) nor complete positivity as Eq. (33) becomes a necessary and sufficient condition for unital channels. 
By assumption the Jamiolkowski state $\tau=\left(T_{1} T_{2} \otimes \mathrm{id}\right)(\omega)$ has rank three. As unital qubit channels are convex combinations of unitary conjugations and $(U \otimes \mathrm{id})(\omega)=$ $\left(\mathrm{id} \otimes U^{T}\right)(\omega)$ we can write

$$
\tau=\left(T_{1} \otimes T_{2}^{T}\right)(\omega),
$$

where $T_{2}^{T}$ is again a unital channel whose Kraus operators are related to those of $T_{2}$ by transposition. It follows from Eq. (47) that the Kraus rank of $T_{1}$ and $T_{2}$ is at most three. Assume now $T_{2}$ has Kraus rank three. Then $\tau \geq \mu\left(T_{1} \otimes \mathrm{id}\right)(\mathbb{1}-\Omega)$ where $\Omega$ is the projector onto a maximally entangled state and $\mu$ is the smallest non-zero eigenvalue of the Jamiolkowski state of $T_{2}$. Thus, if $\left\{\sqrt{p_{i}} U_{i}\right\}$ are the Kraus operators of $T_{1}$ with $\left\{U_{i}\right\}$ orthogonal unitaries and $\left\{p_{i}\right\}$ probabilities, then

$$
\tau \geq \mu\left(\mathbb{1}-\sum_{i} p_{i}\left(U_{i} \otimes \mathbb{1}\right) \Omega\left(U_{i} \otimes \mathbb{1}\right)^{\dagger}\right) .
$$

Since the projectors in the sum are orthogonal, $\tau$ can only be rank deficient if there is only a single term in the sum and $T_{1}$ thus a unitary.

The only remaining possibility is thus a decomposition into two unital channels each of Kraus rank two. In order to rule this out note that in this case the support of $\tau$ equals that of

$$
\mathbb{1}-\sum_{i=1}^{2} p_{i}\left(U_{i} \otimes \mathbb{1}\right) P\left(U_{i} \otimes \mathbb{1}\right)^{\dagger},
$$

where $P$ is now some two-dimensional projector. Denoting by $\psi$ the normalized and maximally entangled null vector of $\tau$ we have to have that $P\left(U_{i} \otimes \mathbb{1}\right)^{\dagger}|\psi\rangle=\left(U_{i} \otimes \mathbb{1}\right)^{\dagger}|\psi\rangle$ so that

$$
P=\sum_{j=1}^{2}\left(U_{j} \otimes \mathbb{1}\right)^{\dagger}|\psi\rangle\langle\psi|\left(U_{j} \otimes \mathbb{1}\right) .
$$

Now we exploit the fact that every basis of orthogonal unitaries $\left\{U_{j}\right\}$ in $\mathcal{M}_{2}$ is essentially equivalent to the Pauli basis in the sense that there are always unitaries $V_{1}, V_{2}$ and phases $e^{i \varphi_{j}}$ such that $U_{j}=V_{1} \sigma_{j} V_{2} e^{i \varphi_{j}}$ [28]. It follows that $U_{1} U_{2}^{\dagger}$ equals $U_{2} U_{1}^{\dagger}$ up to a phase which in turn implies that the expression in Eq. (49) and thus $\tau$ have rank two-contradicting the assumption and therefore concluding the proof.

C. Infinitesimal divisible qubit channels. We will now give a necessary and sufficient criterion for qubit channels to be infinitesimal divisible, formulated in terms of the matrix representation Eq. (32) of the channel's Lorentz normal form (Thm. 18):

Theorem 24 (Characterization of infinitesimal divisible channels). Consider a qubit channel and denote by $s_{\text {min }}$ the smallest singular value of the $\Delta$-block of its Lorentz normal form. The channel is infinitesimal divisible iff one of the following conditions is true

1. The Lorentz normal form is not diagonal.

2. The normal form is diagonal and $\operatorname{rank}(\Delta)<2$. 
3. The normal form is diagonal and

$$
s_{\text {min }}^{2} \geq \operatorname{det} \Delta>0 \text {. }
$$

Proof. We exploit the fact that by Thm. 17 a channel is infinitesimal divisible iff its Lorentz normal form is. If the normal form is not diagonal, then by Eqs. $(34,35)$ it has Kraus rank two or is a product of Kraus rank-two channels which are in turn infinitesimal divisible according to Thm. 19. Similarly, if the normal form is diagonal and $\Delta=\operatorname{diag}(\lambda, 0,0)$ we can again factorize it into Kraus rank-two channels as $\Delta=$ $\operatorname{diag}(1,0,0) \operatorname{diag}(\lambda, 1, \lambda)$. To complete point 2 in the theorem note that the unital channel with $\Delta=0$ is a limit of a Markovian unital channel as $\Delta=\lim _{t \rightarrow \infty} e^{-t} \mathbb{1}$.

Consider now the generic case where the Lorentz normal form is diagonal and $\operatorname{det} T \neq$ 0 . Following Prop. 15 we have that det $T \geq 0$ for every infinitesimal divisible channel. Moreover, by Thm. 16 we can express these channels in terms of products of Markovian channels, which can w.l.o.g. be chosen unital. The latter can in turn be decomposed into even simpler pieces by exploiting the Lie-Trotter formula $\lim _{n \rightarrow \infty}\left(e^{L_{1} / n} e^{L_{2} / n}\right)^{n}=$ $e^{L_{1}+L_{2}}$. In this way every unital Markovian qubit channel can be written as a product of unitaries and unital Kraus rank-two channels with $\Delta=\operatorname{diag}(1, \lambda, \lambda)$ [29]. Note that for these channels we have $s_{\min }^{2}=\operatorname{det} T$. The inequality Eq. (51) follows then from concatenating these channels together with multiplicativity of the determinant and the fact that $s_{\min }\left(\Delta_{1}\right) s_{\min }\left(\Delta_{2}\right) \leq s_{\min }\left(\Delta_{1} \Delta_{2}\right)$.

Let us now show the converse, i.e., that Eq. (51) together with a diagonal Lorentz normal form implies that the channel is infinitesimal divisible. To this end we introduce $\Delta_{t}:=\exp (t \ln \Delta), t \geq 0$ and show that it corresponds to a completely positive unital semigroup if $\Delta$ (chosen positive definite and diagonal) satisfies Eq. (51). Following Eq. (33) we have to show that $\operatorname{tr} \Delta_{t} \leq 1+2 s_{\min }\left(\Delta_{t}\right)$ for complete positivity. Moreover, it suffices to prove this for infinitesimal $t$ since larger times are obtained by concatenation which preserves complete positivity. In leading order we get

$$
\begin{aligned}
\operatorname{tr} \Delta_{t} & =\operatorname{tr}[\mathbb{1}+t \ln \Delta]+O\left(t^{2}\right) \\
& =3+t \ln \operatorname{det} T+O\left(t^{2}\right), \\
1+2 s_{\text {min }}\left(\Delta_{t}\right) & =1+2\left(1+t \ln s_{\text {min }}(\Delta)\right)+O\left(t^{2}\right),
\end{aligned}
$$

from which we obtain

$$
\operatorname{tr} \Delta_{t}-\left[1+2 s_{\min }\left(\Delta_{t}\right)\right]=t\left[\ln \operatorname{det}(\Delta)-\ln s_{\min }^{2}(\Delta)\right] \pm O\left(t^{2}\right),
$$

which is indeed negative for infinitesimal $t$ if $\operatorname{det}(\Delta)<s_{\min }^{2}(\Delta)$. The case of equality is covered by the fact that we can then express $\Delta=\operatorname{diag}\left(\lambda_{1}, \lambda_{2}, \lambda_{1} \lambda_{2}\right)=\operatorname{diag}$ $\left(\lambda_{1}, 1, \lambda_{1}\right) \operatorname{diag}\left(1, \lambda_{2}, \lambda_{2}\right)$ as concatenation of two Kraus rank-two channels.

What remains to discuss is the case of a diagonal normal form with $\Delta=\operatorname{diag}\left(\lambda_{1}, \lambda_{2}, 0\right)$, $\lambda_{i}>0$. Note that channels of zero determinant can be infinitesimal divisible due to the fact that we took the closure in Def. 14. Hence, there must be an infinitesimal divisible channel $T_{\epsilon}$ with non-zero determinant in every $\epsilon$-neighborhood of $T$. If $T$ is unital we can again w.l.o.g. choose $T_{\epsilon}$ to be unital as well. In leading order the $\Delta$-block of $T_{\epsilon}$ has singular values $\lambda_{1}, \lambda_{2}$ and $\epsilon$. For sufficiently small $\epsilon$ this can, however, never satisfy Eq. (51) so that there cannot be an infinitesimal divisible channel with non-zero determinant close to $T$ and thus $T$ itself cannot be infinitesimal divisible. 
Theorem 24 characterizes the set of qubit channels which are solutions of continuous time-dependent master equations for completely positive evolutions. As in the theory of open quantum systems complete positivity is often dropped in the context of timedependent master equations we provide the analogous statement for evolutions which are (locally) merely positivity preserving:

Theorem 25 (Continuous positive evolutions). A qubit channel $T \in \mathfrak{T}^{+}$is infinitesimal divisible within the set $\mathfrak{T}$ of positive trace preserving maps iff it has non-negative determinant.

Proof. By multiplicativity and continuity of the determinant we know that $\operatorname{det} T \geq 0$ is indeed necessary for $T$ to be infinitesimal divisible. In order to prove sufficiency we exploit once again the Lorentz normal form together with Thm. 17 and the fact that the sign of the determinant does not change upon concatenating with Kraus rank-one filtering operations. If the normal form is not diagonal, then $T$ is infinitesimal divisible according to Thm. 24. If the normal form is diagonal and det $T>0$, then the statement follows from the fact that the corresponding unital channel is an element of a positivity preserving semigroup given by $\Delta_{t}=\exp [t \ln \Delta]$. As $\Delta_{t} \leq \mathbb{1}$ for all $t \geq 0$ the corresponding map is always positive. The remaining cases with det $T=0$ are obtained by taking the closure.

\section{Conclusion}

We have mainly addressed two questions: which quantum channels can be broken down into infinitesimal pieces, and which can be expressed as a non-trivial concatenation of other channels at all. This led us to the two notions of infinitesimal divisibility and divisibility respectively. Loosely speaking, the former class corresponds to the set of solutions of time-dependent master equations. However, to make this a strong correspondence continuity of the Liouville operator (at least piecewise) would clearly be desirable. This follows from our analysis only for qubit channels for which a rather exhaustive characterization was possible. For higher dimensions a similar complete classification might be hard to obtain unless one restricts to specific classes like diagonal or quasi-free channels [30].

We find it remarkable that in the vicinity of the ideal channel all types of channels can be found (i.e., indivisible, divisible, not infinitesimal divisible, Markovian, etc.). This is, in fact, what makes the proof of our main structure theorem non-trivial-if all channels close to the identity would be Markovian, it would follow immediately.

Apart from the implications for the theory of open quantum systems and the abstract semigroup structure of the set of quantum channels we can think of applying the techniques and results presented in this work in various contexts.

Renormalization-group transformations for quantum states on a spin chain [31] for instance use concatenations and-in the infrared limit - divisions of quantum channels.

Moreover, when considering quantum channels with a classical output in the sense of the positive operator valued measure (POVM) formalism, then a similar train of thought leads to the notion of clean POVMs which cannot be expressed as a non-trivial concatenation of a quantum channel with a different POVM [19].

Finally, it would be interesting to know whether a concatenation of quantum channels allows for a quantitative estimate of the channel capacity based on the capacities of the constituents which goes beyond the trivial bottleneck-inequality. In this context also the stability of the above introduced notions under tensor products is an interesting problem. 
Acknowledgement. We thank T. Cubitt, J. Eisert and A. Holevo for valuable discussions.

\section{References}

1. Holevo, A.S.: Statistical Structure of Quantum Theory. Springer Lecture Notes in Physics, BerlinHeidelberg-New York: Springer, 2001

2. Horn, R.A.: Z. Wahrscheinlichkeitstheorie Und Verw. Gebiete 8, 219 (1967)

3. Holevo, A.S.: Theor. Probab. Appl. 32, 560 (1986)

4. Denisov, L.V.: Th. Prob. Appl. 33, 392 (1988)

5. Jamiolkowski, A.: Rep. Math. Phys. 3, 275 (1972)

6. Choi, M.D.: Lin. Alg. Appl. 10, 285 (1975)

7. Kraus, K.: States, Effects, and Operations. Berlin-Heidelberg-New York: Springer, 1983

8. Wolf, M.M., Perez-Garcia, D.: Phys. Rev. A 75, 012303 (2007)

9. Lindbald, G.: Commun. Math. Phys. 48, 119 (1976)

10. Gorini, V., Kossakowski, A., Sudarshan, E.C.G.: J. Math. Phys. 17, 821 (1976)

11. Davies, E.B.: Rep. Math. Phys. 17, 249 (1980)

12. Perez-Garcia, D., Wolf, M.M., Petz, D., Ruskai, M.B.: J. Math. Phys. 47, 083506 (2006)

13. Schmidt, W.M.: Diophantine Approximation. Lecture Notes in Math. 785, Berlin-Heidelberg-New York: Springer Verlag, 1980

14. Bhatia, R.: Matrix Analysis. Springer Graduate Texts in Mathematics 169, Berlin-Heidelberg-New York: Springer, 1997

15. Streater, R.F.: Statistical Dynamics. London: Imperial College Press, 1995

16. Wigner, E.P.: Gruppentheorie. Braunschweig: Vieweg 1931; Group Theory. London: Academic Press, 1959

17. Bargmann, V.: J. Math. Phys. 5, 862 (1964)

18. Kadison, R.: Topology 3(supp. 2), 177 (1965)

19. Buscemi, F., D’Ariano, G.M., Keyl, M., Perinotti, P., Werner, R.: J. Math. Phys. 46, 082109 (2005)

20. Nielsen, M.A., Chuang, I.L.: Quantum Computation and Quantum Information. Cambridge: Cambridge University Press, 2000

21. Uhlmann, A.: Rep. Math. Phys. 9, 273 (1976)

22. Stoermer, E.: Acta Math. 110, 233 (1963)

23. King, C., Ruskai, M.B.: IEEE Trans. Info. Theory 47, 192 (2001)

24. Fujiwara, A., Algoet, P.: Phys. Rev. A 59, 3290 (1999)

25. Ruskai, M.B., Szarek, S., Werner, E.: Lin. Alg. Appl. 347, 159 (2002)

26. Gorini, V., Sudarshan, E.C.G.: Commun. Math. Phys. 46, 43 (1976)

27. Verstraete, F., Verschelde, H.: http://arxiv.org/list/quant-ph/0202124, 2002; F. Verstraete, J. Dehaene, B. De Moor.: Phys. Rev. A 64, 010101(R) (2001)

28. Vollbrecht, K.G.H., Werner, R.F.: J. Math. Phys. 41, 6772 (2000)

29. Bacon, D., Childs, A.M., Chuang, I.L., Kempe, J., Leung, D.W., Zhou, X.: Phys. Rev. A 64, 062302 (2001)

30. Eisert, J., Wolf, M.M.: http://arxiv.org/list/quant-ph/0505151, 2005; 'Gaussian quantum channels'. In: Quantum Information with continuous variables of atoms and light, N. Cerf, G. Leuchs, E.S. Polzik (eds.) London: Imperial College Press, 2006

31. Verstraete, F., Cirac, J.I., Latorre, J.I., Rico, E., Wolf, M.M.: Phys. Rev. Lett. 94, 140601 (2005)

32. Wolf, M.M., Eisert, J., Cubitt, T.S., Cirac, J.I.: arXiv: 0711.3172 (2007)

Communicated by M.B. Ruskai 